\title{
Influence of paeoniflorin on intracellular calcium ion concentration in the sphincter of Oddi of hypercholesterolemic rabbits
}

\author{
F. Wang ${ }^{1}$, C.-M. Wang ${ }^{2}$, J.-D. Liu ${ }^{2}$ and Y.-T. Wang ${ }^{2}$ \\ ${ }^{1}$ Department of Physical Examination, \\ The First Affiliated Hospital of Dalian Medical University, Dalian, China \\ ${ }^{2}$ Department of General Surgery, \\ The First Affiliated Hospital of Dalian Medical University, Dalian, China \\ Corresponding author: C.-M. Wang \\ E-mail: changmiaowang@126.com
}

Genet. Mol. Res. 13 (3): 5001-5010 (2014)

Received June 17, 2013

Accepted January 21, 2014

Published July 4, 2014

DOI http://dx.doi.org/10.4238/2014.July.4.15

\begin{abstract}
This study aimed to investigate the influence of hypercholesterolemia $(\mathrm{HC})$ on intracellular calcium ion concentration in the sphincter of Oddi (SO) of rabbits and the influence of paeoniflorin on intracellular calcium ion concentration in the hypercholesterolemic rabbit SO. Sixteen purebred New Zealand rabbits were randomly divided into two groups: the control group and the HC model group (8 rabbits in each group). The control group was fed standard diet. The $\mathrm{HC}$ group was fed standard diet plus cholesterol for a total of 8 weeks to induce and establish the rabbit $\mathrm{HC}$ model. The SO segment of HC rabbits was taken and enzyme treated to obtain SO cells. After primary culture, immunohistochemical analysis was performed. Fluo-3/AM was used to load SO cells, and then intracellular calcium ion concentration was determined by confocal microscopy. Intracellular calcium ion in the SO of the HC group was higher than that of the normal group; intracellular calcium ion in the $\mathrm{HC}$ rabbit $\mathrm{SO}$ of the paeoniflorin group was lower than that of the control group, where the paeoniflorin effect
\end{abstract}


was greater with higher concentrations. High cholesterol caused an increase in intracellular calcium ion concentration in the rabbit SO, and paeoniflorin can reduce intracellular calcium ion concentration in the $\mathrm{HC}$ rabbit $\mathrm{SO}$ in a concentration-dependent manner.

Key words: Paeoniflorin; Cholesterol; Sphincter of Oddi; Intracellular calcium ion concentration

\section{INTRODUCTION}

The sphincter of Oddi (SO) is a sphincter surrounding the common bile duct ampulla and pancreaticobiliary end, with fine-labor division muscular structures, and has four components: common bile duct sphincter, pancreatic duct sphincter, three-part circular smooth muscle fibers of the teat sphincter, and longitudinal muscle bundle. It is the sole portal of biliary tract excretion of bile. Both SO manometry and electrophysiological study results indicate that SO has a characteristic of periodic forward contraction and is synchronous and independent of the duodenum, which is the important foundation of SO physiological function. In recent years, knowledge about SO physiological function has been increasingly thorough, thereby speeding up research on the dynamic changes of SO under pathophysiological conditions.

Sphincter of Oddi dysfunction (SOD) means that SO loses normal relaxation and contraction functions and SO spasm causes increased pressure, resulting in bile and pancreatic juice obstruction at the pancreaticoduodenal junction and thereby generating a series of clinical symptoms such as upper abdominal bile pancreatic pain, postprandial flatulence, choledochectasia, abnormal liver function, or pancreatic enzyme elevation (Chuttani and Carr-Locke, 1993; Lehman and Sherman, 1996). Domestic and foreign studies in recent 20 years have shown that SOD is not only the leading link of the pathogenesis of most biliary system and pancreas diseases, but also the key secondary pathophysiological change in these diseases. In recent years, SOD diagnosis is greatly helped with the implementation of ERCP, SO manometry, ultrasonic examination, radionuclide scintiscanning technique and other diagnosis measures, and it is found that SOD is not a rare disease. However, it is still difficult to obtain a correct diagnosis of SOD. Also, an effective treatment is lacking. With researches focusing more on the relationship of hypercholesterolemia (HC) with SOD, Zhang et al. (2008) used $\mathrm{HC}$ to induce SOD and found that high cholesterol levels could damage the relaxation function of the bile duct and thereby induce SOD. At present, HC rabbit is the only SOD model. Further studies indicate that intracellular calcium overload and abnormal calcium oscillation play an important role in SOD pathogenesis. Our study confirmed that the active component of Chinese herb peony, paeoniflorin, induces relaxation of the rabbit $\mathrm{SO}$ muscle ring in vitro (Luo et al., 2009). We asked then what is its mechanism of action?

Calcium ions are the second messenger present universally in cells. It is found from studies on the relationship of relaxation and contraction of gastrointestinal smooth muscle with the change in intracellular calcium ions, showing that intracellular free calcium ion concentration is closely related to the relaxation and contraction of smooth muscle (Berridge, 2008). Smooth muscle cell contraction and relaxation occur with changes in calcium ion concentration. Extracellular calcium ion influx and release of intracellular calcium stored in the sarcoplasmic reticulum into the cytoplasm jointly cause an increase in intracellular free cal- 
cium ion concentration and result in smooth muscle contraction. Otherwise, they result in smooth muscle relaxation. Since $\mathrm{SO}$ is also a smooth muscle, does paeoniflorin relax SO by regulating SO intracellular calcium ion concentration?

Accordingly, a laser confocal microscope was used to detect SO intracellular calcium ion concentration difference between normal rabbits and $\mathrm{HC}$ rabbits in this experiment. In addition, different concentrations of paeoniflorin were used to load $\mathrm{HC}$ rabbit SO cells, and different concentrations of paeoniflorin were tested to investigate its mechanism of action.

\section{MATERIAL AND METHODS}

\section{Animals}

Purebred male and female New Zealand big ear rabbits (ages ranging from 3 to 4 months old, body weights of 1.5 to $2.0 \mathrm{~kg}$ ) were purchased from the Experimental Animal Center of Dalian Medical University (Animal Certificate No. SCXK(Liao)2008-0002). This study was carried out in strict accordance with the recommendations in the Guide for the Care and Use of Laboratory Animals of the National Institutes of Health. The animal use protocol had been reviewed and approved by the Institutional Animal Care and Use Committee (IACUC) of the First Affiliated Hospital of Dalian Medical University.

\section{Model preparation}

A total of 16 purebred New Zealand rabbits with ages ranging from 2 to 3 months and body weights of 2.0 to $2.5 \mathrm{~kg}$ were randomly divided into two groups: the control group and the experimental group, 8 rabbits in each group. For the control group, the rabbits were fed the standard diet. For the experimental group (HC), the rabbits were fed the standard diet plus cholesterol for 8 weeks. In each week, after $1.0 \mathrm{~g}$ cholesterol was added to the standard diet daily and continuously for 6 days, cholesterol feeding was stopped for 1 day. Before the experiment, serum cholesterol concentration was determined. According to the judgment criteria of the HC model, if the serum total cholesterol level was less than $3.0 \mathrm{mM}$, it was normal. If it was more than $10 \mathrm{mM}$, it met the $\mathrm{HC}$ criteria. The rabbits whose serum total cholesterol was between 3.0 and $10 \mathrm{mM}$ were excluded. Total cholesterol in serum was determined with a cholesterol kit (Shanghai Chemical Reagent Company, China).

\section{Cell extraction}

New Zealand rabbit was anesthetized with chloral hydrate given via intraperitoneal injection. The SO segment was isolated and the nipple part was cut off. Subsequently, the SO segment was placed on a culture dish with a diameter of $3 \mathrm{~cm}$ containing culture medium. The serous membrane and connective tissue were removed under a stereomicroscope to open the SO segment with a longitudinal cut. After removing mucous and connective tissues on the surface, the remaining part was transferred to an ultra-clean room and washed 3 times with PBS and penicillin and streptomycin mixture. The above SO muscle strip was cut into small blocks of about $1-2 \mathrm{~mm}^{3}$ and placed in a $10-\mathrm{mL}$ digestion solution containing $0.1 \%$ collagenase II and $0.01 \%$ soybean trypsin inhibitor. Digestion was carried out at $37^{\circ} \mathrm{C}$ for about $4 \mathrm{~h}$, 
with a shaking every $30 \mathrm{~min}$. It was noted that the tissue blocks became small, edges became indistinct, and the clarified solution became a suspension. After the appearance of a number of fusiform smooth muscle cells, digestion was stopped, and the resulting digestion mixture was collected, filtered with a sieve of $500 \mu \mathrm{m}$ and centrifuged for $5 \mathrm{~min}$ at $1000 \mathrm{rpm}$. After the supernatant was removed, the remaining pellet was resuspended in culture medium. Finally, trypan blue staining was performed to determine cell viability. If cell viability was over $90 \%$, the experimental requirements were met. In addition, the cell concentration was adjusted to 5 x $10^{5}$ cells $/ \mathrm{mL}$.

\section{Cell viability}

First, a 9-mL cell suspension and $1 \mathrm{~mL} \mathrm{0.4 \%} \mathrm{trypan} \mathrm{blue} \mathrm{were} \mathrm{mixed} \mathrm{well.} \mathrm{After} \mathrm{staining}$ for $3 \mathrm{~min}$, a small amount of mixture was placed on a slide and covered with a coverslip. Under a microscope, observations were carried out using several randomly selected fields. Viable cells were not stained with trypan blue. Under a microscope, viable cells were colorless, and non-viable cells were stained blue. If cell viability was over $90 \%$, the experimental requirements were met.

\section{Morphological observation}

Under an inverted microscope, it was found that individual smooth muscle cells became fusiform or taeniform. Cytoplasm was abundant, cytoplasm density was high, cells were opaque, there were multiple cell protrusions, and the nucleus was orbicular-ovate and at the center of cell, and there were multiple plasmosomes. As cell density increased, they were arranged into parallel bundles, and some cells overlapped, showing growth.

\section{Immunohistochemical detection}

Cells were added to a culture dish $(60 \mathrm{~mm})$ containing a sterile coverslip $(18 \times 18 \mathrm{~mm})$ coated with polylysine at a density of $5 \times 10^{5}$ cells $/ \mathrm{mL}$ and placed in a $\mathrm{CO}_{2}$ incubator. After the cells grew into a monolayer, they were taken out, washed with PBS for 5 min, fixed with $95 \%$ alcohol for $30 \mathrm{~min}$, washed again with PBS for $5 \mathrm{~min}$ and air-dried. The cells were blocked with $5 \%$ BSA for $20 \mathrm{~min}$ at room temperature, and the excess liquid was removed. Next, 50 $\mu \mathrm{L}$ diluted (1:50) mouse anti-human $\alpha$-smooth muscle actin monoclonal antibody was added, and the cells were incubated in a humid box at $4^{\circ} \mathrm{C}$ overnight and washed with PBS for 2 min three times. Afterwards, biotinylated goat anti-mouse IgG was added, and the cells were incubated for $20 \mathrm{~min}$ at $37^{\circ} \mathrm{C}$ and washed with PBS for $2 \mathrm{~min}$ three times. After adding the $\mathrm{SABC}$ reagent, the mixture was incubated for $20 \mathrm{~min}$ at $37^{\circ} \mathrm{C}$ and washed with PBS for $5 \mathrm{~min}$ four times. Subsequently, the slides were subjected to DAB developing and mild hematoxylin counterstaining. Afterwards, the slides were air-dried and mounted. The slides were then examined under a microscope.

\section{Primary cell culture}

Six rabbits were respectively sampled from the normal group and the HC group, and primary culture was established at different times. At 3 days after primary culture, it was vis- 
ible under a microscope that $80 \%$ cells grew by adhering to the surface, and cells became long fusiforms and overlapped in peak and valley shape. The culture medium was removed and 1 $\mathrm{mL} 0.25 \%$ trypsin plus $0.02 \%$ EDTA was added. After $30 \mathrm{~s}$ at room temperature, when cells appeared brilliant and gradually rounded under an inverted microscope, $1 \mathrm{~mL}$ DMEM solution containing $20 \%$ fetal bovine serum was added to terminate digestion. The digestion liquid in the culture flask was removed, and $3 \mathrm{~mL}$ complete DMEM was added. The cells were resuspended in culture medium to prepare the cell suspension and added to a 50-mm culture dish coated with polylysine at a ratio of 1:2. The dishes were then placed in an incubator for $24 \mathrm{~h}$, and after cells were attached, they were used for next tests.

\section{Fluo-3/AM loading}

1. Fluo-3/AM loading solution preparation: Fluo-3/AM ( $5 \mathrm{mM})$ in anhydrous DMSO was diluted with D-Hanks solution to obtain a $5-\mu \mathrm{M}$ solution.

2. Fluo-3/AM loading cells: The culture medium was removed and the cells washed twice with D-Hanks solution, and $100 \mu \mathrm{L}$ Fluo-3/AM loading solution was added. Subsequently, the culture dish was protected from light and incubated at $37^{\circ} \mathrm{C}$ for $30 \mathrm{~min}$. The culture dish was washed with D-Hanks solution 3 times to remove excess dye, and a small amount of D-Hanks solution was kept to balance cells. Intracellular calcium ion concentration was determined within 10 min under confocal microscope.

3. Determination: A German Leica TCS SP5 model confocal microscope was used (excitation wavelength: $488 \mathrm{~nm}$; emission wavelength: $526 \mathrm{~nm}$ ). For each specimen, 4 photographs were randomly taken and saved. The images were analyzed by the Image-Pro Plus software to obtain the mean optical density value. Since Fluo-3/AM binds to intracellular calcium ions at a ratio of 1:1, intracellular calcium ion concentration is reported as mean optical density.

\section{Primary culture of HC rabbit cells}

$\mathrm{HC}$ rabbit was sampled, and the $\mathrm{SO}$ was isolated under aseptic conditions. SO cells were extracted by the enzyme digestion method, and primary culture was established and the cells then passaged. Smooth muscle cells of the $3 \mathrm{rd}$ to 5 th passages were digested, and the cell concentration was adjusted to $5 \times 10^{5} / \mathrm{mL}$. The cells were added to four 6-well plates with pre-placed coverslips $(1.8 \times 1.8 \mathrm{~mm})$ and four wells were selected on each 6-well plate. Each 6-well plate was used for one group, and there were a total of 4 groups: the control group (normal medium) and groups with high, moderate or low dose of the paeoniflorin. After incubation for $24 \mathrm{~h}$, cells attached, the medium was removed, and low-sugar DMEM culture liquid without serum was added. The cells were incubated for $24 \mathrm{~h}$ to allow cells to synchronously grow. Subsequently, different concentrations of paeoniflorin were added to load cells for $30 \mathrm{~min}$, and the culture medium was removed. According to the above method, intracellular calcium ion concentration was determined.

\section{Statistical analysis}

The data obtained are reported as means \pm standard deviation, the SPSS13.0 software was used for data analysis, and the $t$-test was used for comparisons between the two groups. $\mathrm{P}$ $<0.05$ indicated a significant difference. 


\section{RESULTS}

\section{Total cholesterol content in rabbit serum}

Before the experiment, total cholesterol contents in serum of both the control group and the $\mathrm{HC}$ group were normal $(<3 \mathrm{mM})$, and there was no significant difference between the two groups $(\mathrm{P}>0.05)$. After cholesterol feeding, total cholesterol content in serum was 28.17 $\pm 2.58 \mathrm{mM}$, substantially higher than $10 \mathrm{mM}$. Compared with pre-experiment and control group values, there were significant differences $(\mathrm{P}<0.05$; Table 1$)$.

Table 1. Total cholesterol content $(\mathrm{mM})$ in rabbit serum.

\begin{tabular}{lcc}
\hline Groups & Before feeding & After feeding \\
\hline Control rabbit & $1.49 \pm 0.23$ & $1.48 \pm 0.2$ \\
Hypercholesterolemic rabbit & $1.48 \pm 0.25$ & $28.17 \pm 2.58$ \\
\hline
\end{tabular}

Cholesterol concentrations in serum, before feeding, of both the control group and the hypercholesterolemic (HC) group were normal, and there was no significant difference $(\mathrm{P}>0.05)$. Compared with before feeding, total cholesterol concentration in serum after feeding was significantly increased $(\mathrm{P}<0.05)$, whereas for the control group, there was no difference.

\section{SO cell morphology observation}

As freshly isolated SO cells were in the contraction status, the cells appeared brilliant and nearly rounded under the inverted microscope. In the incubator, the cells were attached within about $48 \mathrm{~h}$. Under the inverted microscope, individual smooth muscle cells appeared fusiform or taeniform, and some cells showed small bifurcations at the two ends, with the cytoplasm being abundant and very dense. Cells were opaque, the nucleus was orbicular-ovate and at the center of cell, and there were multiple plasmosomes. As cells grew densely, they were arranged into bundles in parallel, and some cells overlapped (Figure 1).

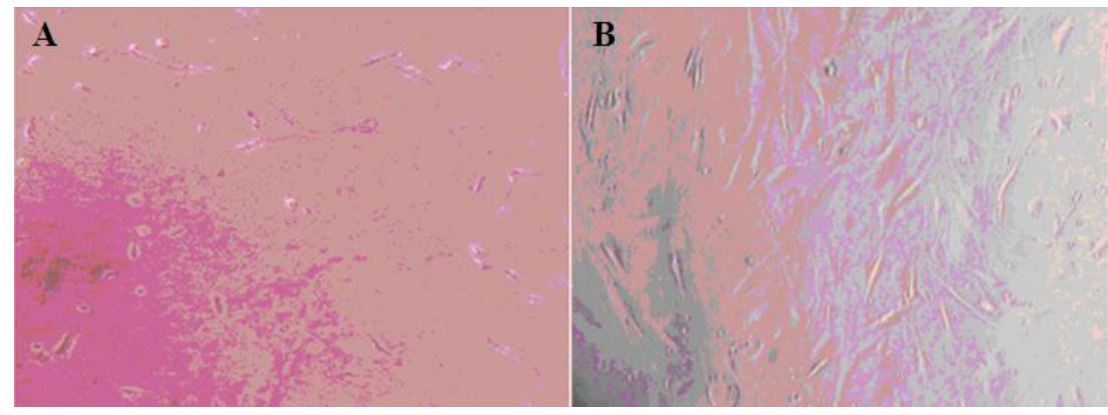

Figure 1. A. Adhesive sphincter of Oddi (SO) cells after primary culture for 1 day (100X). B. Well-grown SO cells after culture (100X).

\section{Immunohistochemical identification of SO cells}

There was specific $\alpha$-actin expression in cells, and immune reaction products appeared 
yellowish-brown. Under a microscope, a number of brown fiber filaments parallel to the long axis of cell were visible in the cytoplasm (Figure 2A), apparently $\alpha$-actin. More than $>98 \%$ of cells were positive for $\alpha$-actin.

\section{HC and calcium ion concentration}

Calcium ion concentration is reported as the mean optical density of SO cells determined after fluorescence staining (Figure 2B). The results showed that the mean intracellular optical density value of the $\mathrm{HC}$ group was higher than that of the normal group $(0.330 \pm 0.285 \mathrm{vs}$ $0.073 \pm 0.009)$, and there was a significant difference between the two groups $(\mathrm{P}<0.05$; Table 2$)$.

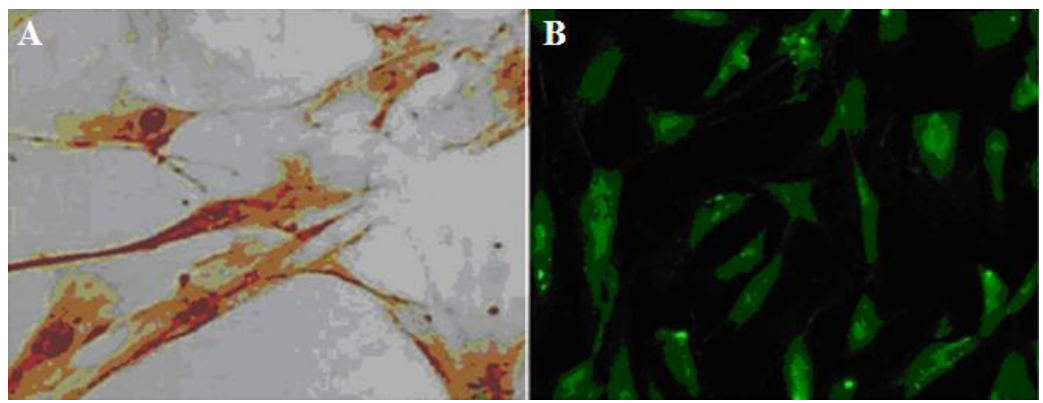

Figure 2. A. Sphincter of Oddi (SO) cells are positive for $\alpha$-actin (200X). B. SO cells stained by Fluo-3/AM (calcium fluorescence probe) (200X).

Table 2. Mean intracellular optical density values of normal and hypercholesterolemic (HC) rabbits.

\begin{tabular}{lcc}
\hline Groups & Mean value & Standard deviation \\
\hline Control & 0.073 & 0.009 \\
HC & 0.330 & 0.285 \\
\hline
\end{tabular}

The mean intracellular optical density value of the $\mathrm{HC}$ group was significantly higher than that of the normal group $(\mathrm{P}<0.05)$.

\section{Paeoniflorin concentration and calcium ion concentration}

SO cells were incubated with medium containing high-, moderate- and low-concentration paeoniflorin and normal medium, and the mean intracellular optical density values were determined under a laser confocal microscope. The results showed that paeoniflorin reduced the mean intracellular optical density value of SO in a concentration-dependent manner (Table 3).

\begin{tabular}{lcc} 
Table 3. Mean intracellular optical density value. \\
\\
\hline Groups & Mean value & Standard deviation \\
\hline Control & 0.320 & 0.030 \\
High-concentration paeoniflorin & 0.142 & 0.030 \\
Moderate-concentration paeoniflorin & 0.209 & 0.031 \\
Low-concentration paeoniflorin & 0.276 & 0.025 \\
\hline
\end{tabular}

Paeoniflorin can reduce the mean intracellular optical density value of SO and the decrease extent of the mean intracellular optical density value increased with an increase of the concentration in serum. 


\section{DISCUSSION}

$\mathrm{SO}$ is located at the junction where the pancreatic duct converges with the duodenum. As the most important anatomic structure of the biliopancreatic junction, it is a sphincter with fine-labor division muscular structures, independent of the duodenum smooth muscle. SO generates coordinated relaxation and contraction motions under the common regulation of nerves and hormones and plays an important role in controlling the flow of bile and pancreatic juice and maintaining biliary system pressure.

SOD treatment is mainly based on expanding SO to reduce the resistance of SO. SOD treatment methods include drug treatment, ES (endoscopic sphincterotomy) and surgical treatment. Surgical treatment has been replaced with ES. At present, ES is an effective treatment method, but there is a high incidence of postoperative pancreatitis and other complications. According to statistics, the incidence rate of postoperative complications of ES is 2.3 to $9.8 \%$ (Vitton et al., 2012).

So far, there is still no effective drug for SOD treatment because a specific and longacting drug without adverse reactions for SOD treatment has not been found. Ineffective drugs include nitrate and calcium channel blockers (Vitton et al., 2008), erythromycin (Wehrmann et al., 1996), carnitine toxin (Wehrmann et al., 1998; Sand et al., 1998), and somatostatin (Fazel et al., 2002). Recently, Vitton et al. (2012) proved that trimebutine could relieve pain generated by SOD, and that trimebutine had the same success rate as ES. This drug could replace ES to treat SOD. In recent years, we used traditional Chinese medicine, the Chinese herb peony and its active component paeoniflorin, to treat SOD and proved that in vitro, paeoniflorin has a relaxation effect on muscle ring of $\mathrm{HC}$ rabbit SO, but the specific mechanism needed to be further studied.

HC can induce rabbit SO dysfunction. Szilvassy et al. $(1997,1998)$ were the first to report a case of high cholesterol and hypertriglyceridemia complicated with SOD. In 2000, Wei et al. successfully established a rabbit SOD model by feeding cholesterol to New Zealand rabbits and proposed that high cholesterol induces SOD. Also, it was found that high cholesterol could cause rabbit SO pressure increase and relaxation function damage, indicating that high cholesterol could cause relaxation and contraction function abnormalities of SO. Zhang et al. (2008) found that HC could increase SO tension and decrease sphincter relaxation function in vitro. Furthermore, laser confocal microscopy showed that the intracellular calcium ion concentration of $\mathrm{HC}$ rabbit SO was increased, and that intracellular calcium overload and calcium oscillation abnormality played an important role in SOD pathogenesis.

$\mathrm{SO}$ is also a smooth muscle. Although SO relaxation and contraction mechanism is still not very clear, there have been more in-depth studies on the relaxation and contraction mechanism of vascular smooth muscle (McDonald et al., 1994; Ares et al., 2000; Hill et al., 2001; Berridge, 2008), which is worth referring to. For multiple relaxation and contraction mechanisms of vascular smooth muscle, the relaxation and contraction of smooth muscle are regulated by influencing intracellular calcium ion metabolism. As the coupling factor of nervous excitation and muscle contraction, calcium ions are an important second messenger, playing an important role in the muscular relaxation and contraction mechanism. In the quiescent condition, calcium ion concentration in the extracellular fluid is more than about 20,000 times the intracellular free calcium ion concentration. When cells are stimulated, cell membrane depolarization causes opening of voltage-dependent calcium channels (mainly L type and $\mathrm{T}$ type) to result in extracellular calcium influx. At the same time, calcium ions are released from intracellular stores into cytoplasm. With this joint action, the calcium ion concen- 
tration is increased, which causes smooth muscle contraction. The former is the main calcium ion source, its release causes calcium ion to rapidly increase to the peak. Afterwards, calcium ions in the cytoplasm are transported outside the cells or into the sarcoplasmic reticulum by calcium pumps on the cell membrane and sarcoplasmic reticulum $\left(\mathrm{Ca}^{2+}-\mathrm{H}\right.$-ATPase $)$ as well as $\mathrm{Na}^{+} / \mathrm{Ca}^{2+}$ reverse concentration gradient on cell membrane, which reduces intracellular calcium ion concentration, causing muscle relaxation. Therefore, calcium ions play an important role in the regulation of smooth muscle relaxation and contraction (Jiang and Stephens, 1994).

In this study, a rabbit HC model was established by feeding cholesterol to New Zealand rabbits, and SO intracellular calcium ion concentration was then determined. The results showed that SO intracellular calcium ion concentration of the HC group was higher than that of the normal group, which was in line with previous studies (Zhang et al., 2008). It is inferred that this is possibly the pathological basis of SO spasm and provides certain evidence for HC inducing SO tension changes and SOD. At the same time, this provides a theoretical basis for the prevention and treatment of clinical SOD.

Radix Paeoniae Alba is the dry root of a ranunculaceous plant without external bark, and it is slightly, bitter and acid. It is a traditional Chinese medicine that is used for nourishing the blood and liver, relieving pains and astringing Yin and absorbing sweat. Previous studies have suggested that Radix Paeoniae Alba and its extract paeoniflorin have spasmolytic and smooth muscle relaxation effects. Our previous study (Luo et al., 2009) confirmed that in vitro, paeoniflorin could also relax HC rabbit SO muscle rings. Chen et al. (2002) proved that paeoniflorin inhibited tetanic contractions of vas deferens, epididymis and prostate smooth muscle of rats, inhibited in vitro intestine and in vivo stomach movement of cavies and rats, as well as uterine smooth muscle of rats, and could antagonize oxytocin-induced contraction. Also, paeoniflorin showed synergism with methanol extract of licorice (FM100).

In this study, paeoniflorin was used to load $\mathrm{HC}$ rabbit SO cells, and the results showed that paeoniflorin reduced the intracellular calcium ion concentration of $\mathrm{HC}$ rabbit $\mathrm{SO}$ in a concentration-dependent manner. Our recent study (Wang et al., 2014) confirmed that in vitro, paeoniflorin can also relax $\mathrm{HC}$ rabbit $\mathrm{SO}$ muscle rings. It is indicated that paeoniflorin relaxes rabbit $\mathrm{SO}$ possibly by reducing intracellular calcium ion concentration of $\mathrm{HC}$ rabbit $\mathrm{SO}$, which provides a theoretical basis for treating SOD with the Chinese herb peony. Further studies are needed to elucidate the mechanism of action of paeoniflorin in reducing intracellular calcium ion concentration of $\mathrm{HC}$ rabbit $\mathrm{SO}$.

\section{REFERENCES}

Ares MP, Porn-Ares MI, Moses S, Thyberg J, et al. (2000). 7Beta-hydroxycholesterol induces $\mathrm{Ca}^{2+}$ oscillations, MAP kinase activation and apoptosis in human aortic smooth muscle cells. Atherosclerosis 153: 23-35.

Berridge MJ (2008). Smooth muscle cell calcium activation mechanisms. J. Physiol. 586: 5047-5061.

Chen YF, Lin YT, Tan TW and Tsai HY (2002). Effects of veratrine and paeoniflorin on isolated mouse vas deferens. Phytomedicine 9: 296-301.

Chuttani R and Carr-Locke DL (1993). Pathophysiology of the sphincter of Oddi. Surg. Clin. North Am. 73: 1311-1322.

Fazel A, Li SC and Burton FR (2002). Octreotide relaxes the hypertensive sphincter of Oddi: pathophysiological and therapeutic implications. Am. J. Gastroenterol. 97: 612-616.

Hill MA, Zou H, Potocnik SJ, Gerald AM, et al. (2001). Transduction in smooth muscle invited review: arteriolar smooth muscle mechanotransduction: $\mathrm{Ca}^{2+}$ signaling pathways underlying myogenic reactivity. J. Appl. Physiol. 91: 973-983.

Jiang H and Stephens NL (1994). Calcium and smooth muscle contraction. Mol. Cell Biochem. 135: 1-9.

Lehman GA and Sherman S (1996). Sphincter of Oddi dysfunction. Int. J. Pancreatol. 20: 11-25. 
Luo JR, Wang CM, Fu L and Sun YP (2009). Effects of paeoniflorin on contractile activity of rabbit sphincter of Oddi muscle rings in vitro. J. Dalian Med. Univ. 31: 668-671.

McDonald TF, Pelzer S, Trautwein W and Pelzer DJ (1994). Regulation and modulation of calcium channels in cardiac, skeletal, and smooth muscle cells. Physiol. Rev. 74: 365-507.

Sand J, Nordback I, Arvola P, Porsti I, et al. (1998). Effects of botulinum toxin A on the sphincter of Oddi: an in vivo and in vitro study. Gut 42: 507-510.

Szilvassy Z, Nagy I, Madacsy L, Hajnal F, et al. (1997). Beneficial effect of lovastatin on sphincter of Oddi dyskinesia in hypercholesterolemia and hypertriglyceridemia. Am. J. Gastroenterol. 92: 900-902.

Szilvassy Z, Sari R, Nemeth J, Nagy I, et al. (1998). Improvement of nitrergic relaxation by farnesol of the sphincter of Oddi from hypercholesterolaemic rabbits. Eur. J. Pharmacol. 353: 75-78.

Vitton V, Delpy R, Gasmi M, Lesavre N, et al. (2008). Is endoscopic sphincterotomy avoidable in patients with sphincter of Oddi dysfunction? Eur. J. Gastroenterol. Hepatol. 20: 15-21.

Vitton V, Ezzedine S, Gonzalez JM, Gasmi M, et al. (2012). Medical treatment for sphincter of oddi dysfunction: can it replace endoscopic sphincterotomy? World J. Gastroenterol. 18: 1610-1615.

Wang F, Wang CM, Luo JR and Qi QH (2014). Fffects of paeoniflorin on the sphincter of Oddi (SO) rings of hypercholesterolemia (hypercholesterolemia, HC) rabbits in vitro. China Health Care Nutr. 24: 456-457.

Wehrmann T, Pfeltzer C and Caspary WF (1996). Effect of erythromycin on human biliary motility. Aliment. Pharmacol. Ther. 10: 421-426.

Wehrmann T, Seifert H, Seipp M, Lembcke B, et al. (1998). Endoscopic injection of botulinum toxin for biliary sphincter of Oddi dysfunction. Endoscopy 30: 702-707.

Wei JG, Wang YC, Du F and Yu HJ (2000). Dynamic and ultrastructural study of sphincter of Oddi in early-stage cholelithiasis in rabbits with hypercholesterolemia. World J. Gastroenterol. 6: 102-106.

Zhang XY, Cui GB, Ma KJ, Wang S, et al. (2008). Sphincter of Oddi dysfunction in hypercholesterolemic rabbits. Eur. J. Gastroenterol. Hepatol. 20: 202-208. 